\title{
Powdery Mildew Resistance in Gerbera: Mode of Inheritance, Quantitative Trait Locus Identification, and Resistance Responses
}

\author{
Xiaohe Song \\ State Key Laboratory of Crop Stress Biology in Arid Areas, College of Plant Protection, Northwest \\ A\&F University, Yangling, Shaanxi 712100, China; Gulf Coast Research and Education Center, \\ Environmental Horticulture Department, University of Florida/IFAS, 14625 County Road 672, \\ Wimauma, FL 33598 \\ Zhanao Deng ${ }^{1}$ \\ University of Florida/IFAS, Environmental Horticulture Department, Gulf Coast Research \\ and Education Center, 14625 County Road 672, Wimauma, FL 33598
}

\begin{abstract}
AdDitional INDEX words. disease resistance, genetics, genetic mapping, molecular marker, Podosphaera xanthii, quantitative trait, sporulation

Aвstract. Gerbera (Gerbera hybrida) is an important floricultural crop in the United States and worldwide. Powdery mildew (PM) caused by Podosphaera xanthii is the most common and destructive disease in gerbera production and landscape use. Gerbera breeding line UFGE 31-19 is one of the few sources of resistance to PM in gerbera and has contributed its resistance to new gerbera cultivars. To determine the mode of inheritance for PM resistance in UFGE 31-19, one of its PM-resistant (PM-R) progeny, UFGE 4033, was crossed with PM-susceptible (PM-S) cultivar, Sunburst Snow White, and their progeny were evaluated for PM severity. Distribution of PM severity ratings among the progeny was continuous but with two peaks, suggesting that the PM resistance in UFGE 4033 and UFGE 31-19 is a quantitative trait, likely controlled by major genes. Bulked segregant analysis (BSA) identified 17 molecular markers present in UFGE 4033 and the PM-R bulk but absent in 'Sunburst Snow White' and the PM-S bulk. Eleven of the molecular markers were mapped to one genetic linkage group, and two regions on this linkage group together explained $71.1 \%$ of the phenotypic (PM severity rating) variance in the segregating population. It was proposed that the two regions be named $R p x 1$ and $R p x 2$ (resistance to $P$. xanthii). Conidia of $P$. xanthii inoculated on the leaf surface of UFGE 4033 germinated, formed secondary germ tubes, and formed appressoria at high percentages, similar to those on the leaf surface of 'Sunburst Snow White'. However, $P$. xanthii hyphae branched significantly less, were significantly shorter, and produced substantially fewer conidia on the leaf surface of UFGE 4033 and its PM-R progeny than on the leaf surface of 'Sunburst Snow White'. These results should provide a sound foundation for use of UFGE 31-19 and progeny UFGE 4033 in gerbera disease resistance breeding and facilitate further investigation and understanding of the genetic bases of $\mathrm{PM}$ resistance in gerbera.
\end{abstract}

Cultivated gerberas are diploids resulting from artificial crosses between Gerbera jamesonii and Gerbera viridifolia (Hansen, 1999), both with $2 n=2 x=50$ chromosomes. Because of their hybrid origin, cultivated gerberas have been given the provisional name Gerbera hybrida (Hansen, 1999). Gerberas are grown mainly as cut flowers and flowering potted plants, but also as patio, garden, and landscape plants. They are valued for their unique, attractive flower forms and bright flower colors and have been a very important crop in the global floriculture trade. Cut gerberas in the Dutch auction alone were worth $€ 100$ million to $€ 125$ million annually and are the fifth most valuable

Received for publication 30 July 2013. Accepted for publication 3 Sept. 2013 We thank the University of Florida/IFAS and the Fred C. Gloeckner Foundation, Inc. for financial support, J. Jones, G. Bowman, R. Kelly, and N. West for excellent technical assistance, and AgriStarts, Inc. for providing gerbera tissue culture liners. X. Song was financially supported, in part, by the 111 Project from the Ministry of Education of China (B07049). Our sincere appreciation goes to Dr. Jinguo Hu and Ms. Lisa Taylor at the USDA/ARS Western Regional Plant Introduction Station, Pullman, WA for their guidance and help with TRAP marker analysis. Drs. "Jake" Henny, Vance Whitaker, and Sameul Hutton reviewed this manuscript and provided valuable comments and suggestions, for which we are very grateful.

${ }^{1}$ Corresponding author. E-mail: zdeng@ufl.edu. floral crop (Evans, 2009; Teeri et al., 2006). In the United States, the annual wholesale value of cut gerberas approaches $\$ 35$ million, with California being the leading producer of cut gerberas (U.S. Department of Agriculture, 2013). Significant potted gerbera production also exists in Texas, California, Illinois, Florida, and Michigan (Granke et al., 2012).

Gerbera breeding is active in numerous countries, especially the Netherlands, Germany, Israel, United States, Canada, Japan, South Korea, China, and Brazil (Chung et al., 2001; Deng and Harbaugh, 2010; Kloos et al., 2005; Mata et al., 2009; Rogers and Tjia, 1990). A very important objective in gerbera breeding is to improve plant resistance to major fungal diseases, particularly PM (Deng and Harbaugh 2010; Kloos et al., 2005).

PM on gerbera is caused mainly by Podosphaera (Sphaerotheca) xanthii (syn. Podosphaera fusca) (Braun et al., 2002; Granke et al., 2012; Kloos et al., 2005; Moyer and Peres, 2012). The fungus forms a network of hyphae over the plant surface, and the leaves and flowers eventually become covered with white, talcum-like colonies (Granke et al., 2012), making plants or cut flowers unsightly and unmarketable. PM has been considered to be the most common and destructive gerbera disease in commercial production and landscape use (Channel, 2005; Granke et al., 2012; Hausbeck, 2004; Hausbeck et al., 
2002). Chemical control has been the primary tool for managing PM in commercial gerbera production. Growers often have to apply fungicides on a weekly basis, which leads to significant increases in production costs and difficulties in crop production. Few management options are available for controlling PM on gerberas in the landscape.

The development and use of PM-R cultivars has helped in controlling PM more effectively, reducing the need for pesticide use, and/or lowering production costs in major crops (Jørgensen, 1994; Li et al., 2005; Miedaner and Flath, 2007). It is expected that PM-R gerbera cultivars may bring similar benefits to growers and consumers (Hausbeck, 2004). So far, only a limited number of sources of PM resistance have been identified in commercial gerbera cultivars or breeding lines. The cut flower gerbera cultivar Terrafame showed significantly less PM than 10 other commercial cut flower cultivars when evaluated in Michigan (Hausbeck et al., 2002). 'Festival SemiDouble Orange', a pot type, was moderately resistant to PM and had only $40 \%$ to $64 \%$ of foliage infected, whereas other gerbera cultivars in the study had $81 \%$ to $100 \%$ of leaves infected with PM (Hausbeck, 2004). In field and greenhouse performance trials of gerbera breeding lines in North Carolina in 1994 and 1995, Kloos et al. (2005) identified two gerbera plants (plants 176 and 214, of unknown parentage) that were highly resistant to $P M$ caused by $P$. xanthii. Information about the use of these sources of $\mathrm{PM}$ resistance in gerbera breeding is not available. In 2001-03, we screened some 2000 gerbera breeding lines under natural but high PM disease pressure in West Central Florida. Seeds of these gerberas were donated by Sunshine State Carnations, Inc. (Hobe Sound, FL), and the parental plants of these gerbera seeds had been naturally "screened" under mildew disease pressure at the grower's production site. Out of these breeding lines, two plants (UFGE 31-19 and UFGE 5-23) were found highly resistant to PM. The PM resistance in UFGE 31-19 has been incorporated into two new gerbera cultivars (Deng and Harbaugh, 2010, 2013), and the new cultivars have shown good PM resistance in greenhouse and garden trials in Florida, Georgia, Illinois, Ohio, and Texas (J. Mazat and K. Roethle, personal communications). Efforts are being made to transfer the PM resistance from UFGE 31-19 into gerbera cultivars with other flower forms (semi double and full double), flower colors (yellow, red, pink, fuchsia, etc.), or other uses (pot or garden type and cut flower).

Kloos et al. (2005) were the first to investigate the mode of inheritance of PM resistance in gerbera. On the basis of segregation of PM resistance in $\mathrm{F}_{1}, \mathrm{~F}_{2}$, and backcross populations, the authors proposed that a dominant allele, Pmrl (powdery mildew-resistant), determined the PM resistance in plants 176 and 214. The Pmrl allele exhibited significant variability in dominance or expressivity, especially in progeny where one parent was from a highly PM-S background (Kloos et al., 2005). The authors suspected that modifying genes might have some effect on the level of PM resistance or susceptibility in gerbera. No information is available in the literature regarding the resistance mechanisms of PM resistance in gerbera. In this study, we investigated the mode of inheritance for the PM resistance from UFGE 31-19 and characterized responses of gerberas carrying the PM resistance to inoculated PM spores.

\section{Materials and Methods}

Parents And their PM resistance. Gerbera breeding lines UFGE 31-19 and UFGE 4033 have proven to be highly resistant to PM, whereas gerbera breeding line UFGE 35-4 and the commercial cultivar Sunburst Snow White have been highly susceptible. When UFGE 31-19 was crossed with UFGE 35-4 in Spring 2003, four out of 52 progenies showed high levels of PM resistance similar to UFGE 31-19's, whereas the rest were susceptible like UFGE 35-4. UFGE 4033 was a PM-R F1 progeny from the cross between UFGE 31-19 and UFGE 35-4 and has been used as a breeding parent in the University of Florida gerbera breeding program. The plant has contributed PM resistance to new gerbera cultivars UFGE 7015 and UFGE 7031 (Deng and Harbaugh, 2010, 2013). UFGE 31-19 and UFGE 4033 were likely heterozygous for genes conferring PM resistance, whereas UFGE 35-4 and 'Sunburst Snow White' were likely homozygous for genes conferring PM susceptibility.

Segregating populations and GROWING CONDITIONS. To study the inheritance of PM resistance originating from UFGE 31-19, UFGE 4033 was crossed reciprocally (cross 7-4 and 7-157) with 'Sunburst Snow White'. The crosses were made in Wimauma, FL, in Spring 2007. UFGE 4033 was the female parent in cross 7-4 and the male parent in cross 7-157.

Plants of 'Sunburst Snow White' were grown from tissue culture liners produced by AgriStarts, Inc. (Apopka, FL); plants of UFGE 4033 were propagated by crown division of mature stock as described by Rogers and Tjia (1990). Parental plants were grown in plastic containers ( 15 or $20 \mathrm{~cm}$ in diameter) filled with soilless potting mix and maintained in a greenhouse in Wimauma, FL. The potting mix was Fafard 3B (Conrad Fafard, Agawam, MA) and was amended with the controlled-release $15 \mathrm{~N}-3.9 \mathrm{P}-10 \mathrm{~K}$ fertilizer Osmocote (Scotts, Marysville, OH) and the trace element fertilizer MicroMax (Scotts) as described by Deng and Harbaugh (2010). Plants were irrigated through drip tubes. The greenhouse was covered with an aluminum shading cloth $(\approx 30 \%$ shading); temperature in the greenhouse was maintained between $18^{\circ} \mathrm{C}$ (night) and $32^{\circ} \mathrm{C}$ (day); additional photoperiodic lighting (about $5 \mu \mathrm{mol} \cdot \mathrm{m}^{-2} \cdot \mathrm{s}^{-1}, 2 \mathrm{~h}$ ) was provided with incandescent light bulbs between mid-September and midMarch each year to achieve at least $12 \mathrm{~h}$ of light.

For crosses, fresh pollen was collected from the male parent and applied directly onto mature stigmas of the female parent using a pencil eraser. Mature seeds were harvested 30-40 d after pollination, dried at $37^{\circ} \mathrm{C}$ for 2 to $4 \mathrm{~d}$, and stored in a cool room $\left(\approx 10^{\circ} \mathrm{C}\right)$. Stored seeds were germinated in a superfine germinating mix (Conrad Fafard), as described by Deng and Harbaugh (2010). Progeny plants were grown in the same manner as described above for parental plants. When needed, progeny plants were propagated asexually through crown division.

Phenotyping Progeny for PM resistance. The cross 7-4 was chosen for detailed PM resistance phenotyping and molecular marker analysis. Three rounds of PM evaluation were performed to determine the PM resistance phenotype for progeny of this cross (hereafter referred to as population 7-4).

In the first round of evaluation, young plants of population 7-4 as well as UFGE 4033 and 'Sunburst Snow White' were grown in Todd planter trays (model 32A; Speedling, Sun City, FL) with cells of $7.6 \times 7.6 \times 7.6 \mathrm{~cm}$. Those trays of plants were kept in an isolated growth room. When plants were $\approx 2$ months old and had developed five to eight leaves (7 Jan. 2011), the plants were inoculated with PM spores using a settling tower method described by Ellwood et al. (2007). Inoculum for this trial was produced on another group of 'Sunburst Snow White' plants grown in a greenhouse and infected with naturally occurring PM. Plants to be inoculated were enclosed inside 
a cardboard box (settling tower, $\approx 60 \mathrm{~cm}$ tall) with an open top and bottom. PM spores were gently dislodged off 'Sunburst Snow White' leaves, using a fine brush, above the top of the cardboard box, and allowed $20 \mathrm{~min}$ to settle onto the young gerbera plants. Inoculated plants were kept at $22{ }^{\circ} \mathrm{C}$ under $85 \%$ to $95 \%$ relative humidity $(\mathrm{RH})$ in the dark for $24 \mathrm{~h}$ and then placed in a growth room. Conditions in the growth room included temperatures between $20{ }^{\circ} \mathrm{C}$ (night) and $24{ }^{\circ} \mathrm{C}$ (day), $14 \mathrm{~h}$ light $/ 10 \mathrm{~h}$ dark, light level $\approx 160 \mu \mathrm{mol} \cdot \mathrm{m}^{-2} \cdot \mathrm{s}^{-1}$, and $\mathrm{RH}$ between $40 \%$ and $75 \%$. Development of PM on the inoculated plants was monitored weekly. On 22 Feb. 2011, inoculated progenies were evaluated for PM severity on a scale of 1 to 10 as described by Hausbeck et al. (2002) with minor modifications where $1=$ no disease, $2=1 \%$ to $10 \%, 3=11 \%$ to $20 \%, 4=$ $21 \%$ to $30 \%, 5=31 \%$ to $40 \%, 6=41 \%$ to $50 \%, 7=51 \%$ to $60 \%$, $8=61 \%$ to $70 \%, 9=71 \%$ to $80 \%$, and $10=81 \%$ to $100 \%$ of leaf area showing PM. The resulting PM severity ratings were referred to as PM-0211.

After the evaluation, leaves were removed from plants; the remaining crown and root ball of each progeny was repotted in 20-cm-diameter plastic containers filled with potting mix as described above and sprayed with the fungicide Pipron (SePRO Corp., Carmel, IN). In early Apr. 2011, the repotted plants were moved to an open-sided shade house ( $\approx 40 \%$ shading) where $\approx 500$ other gerbera stock plants were grown. Their newly emerged leaves were exposed to the natural PM disease pressure in the shade house. PM development was monitored regularly beginning in May 2011. In July, PM severity on the progeny and the parental plants was rated as described above using the same scale as above. The PM severity ratings from this evaluation were referred to as PM-0711.

After the second round of evaluation, existing leaves were removed; each progeny and parental plant was divided into two by crown division, repotted as described above, and sprayed with the fungicide Pipron. The two sets of plants were separated into two blocks and randomly placed in each block. Newly emerged leaves were exposed to natural PM pressure in the same shade house used in the second round of evaluation. PM severity on each plant was rated as described above on $3,11,19$, and 27 Jan. 2012. The eight ratings were averaged by progeny to obtain the PM resistance phenotyping data set PM-0112.

BulKed SEgRegant ANALYSIS. On the basis of the phenotyping data from the above PM evaluations, the eight most resistant and eight most susceptible progeny of population 7-4 were selected to make the PM-R and the PM-S DNA bulks, respectively, as described by Michelmore et al. (1991). Genomic DNA was extracted from healthy, fresh leaves of each progeny using the cetyltrimethyl ammonium bromide protocol of Doyle and Doyle (1987) with minor modifications. DNA concentration of samples was adjusted to $20 \mathrm{ng} \cdot \mu \mathrm{L}^{-1}$. An equal amount of genomic DNA from each progeny of the respective bulks was combined. The two DNA bulks and the genomic DNA of UFGE 4033 and 'Sunburst Snow White' were run simultaneously in polymerase chain reaction (PCR) amplification and polyacrylamide gel electrophoresis (PAGE) (refer to below) to identify DNA bands (or fragments) that were present in both UFGE 4033 and the PM-R bulk but absent in 'Sunburst Snow White' and the PM-S bulk. These DNA bands were considered as molecular markers putatively linked with the PM resistance in UFGE 4033.

MoleCUlar MARKER ANALYSIS. Three molecular marker systems, including the resistance gene candidate (RGC), the simple sequence repeat (SSR, or microsatellite), and the target region amplification polymorphism (TRAP) marker system, were used in the aforementioned BSA to identify molecular markers associated with PM resistance. The RGC markers were designed from gerbera genomic sequences that were cloned by PCR amplification with $R$-gene-derived degenerate primers and showed high levels of similarity to the plant nucleotide bindingsite leucine-rich repeat (NB-LRR) class of disease resistance genes (Song et al., 2012). The SSR markers were developed from gerbera expressed sequence tags (ESTs) in the GenBank (Gong and Deng, 2010). RGC and SSR markers were first screened with UFGE 4033 and 'Sunburst Snow White' for the presence of polymorphism between the two parental gerberas and between the PM-R and the PM-S bulks. Primer sequences, PCR amplification parameters, and restriction enzyme digestion for RGC markers were the same as Song et al. (2012). Primer sequences, PCR amplification parameters, and PAGE conditions used for SSR marker analysis were as described by Gong and Deng (2010).

TRAP marker analysis was carried out as described by $\mathrm{Hu}$ and Vick (2003) and Song et al. (2012). In this marker system, one fixed primer was paired with two arbitrary primers in each PCR, and amplified DNA fragments were detected by the infrared (IR) dye attached to the arbitrary primer (Hu and Vick, 2003). Four arbitrary primers (Ga5, Odd15, Sa12, and TRAP13) were selected out of 32 primers based on their reliability in PCR amplification and showed power to reveal genetic polymorphisms in plants (Hu et al., 2005). Arbitrary primers were $5^{\prime}$-endlabeled with either the IR dye IRD 700 or IRD 800. Their sequences were as follows: 1) Ga5-IR800: GGAACCAAACA CATGAAGA; 2) Odd15-IR700: GCGAGGATGCTA CTGGTT; 3) Sa12-IR700: TTCTAGGTAATCCAACAA, and 4) TRAP13-IR800: GCGCGATGATAAATTATC. Fixed primers used included 30 primers from 15 gerbera RGCs (Song et al., 2012), 99 from 99 SSR-containing gerbera ESTs (Gong and Deng, 2010), and 32 degenerate primers designed from the conserved amino acid motifs of plant NB-LRR class $R$ genes (Collins et al., 1998; Deng et al., 2000).

PCR reactions for TRAP marker development were performed in a $10-\mu \mathrm{L}$ volume, containing $1 \mu \mathrm{L}$ of $10 \times$ PCR reaction buffer (New England BioLabs, Ipswich, MA), 0.6 $\mu \mathrm{L}$ of $25 \mathrm{mM} \mathrm{MgCl}_{2}, 0.8 \mu \mathrm{L}$ of $2.5 \mathrm{~mm}$ dNTPs, $0.2 \mathrm{pmol}$ each of two arbitrary primers labeled respectively with IRD-800 and IRD 700, 10 pmol of one fixed primer (Eurofins MWG Operon, Huntsville, AL), 1 unit of Taq DNA polymerase (New England BioLabs), and $30 \mathrm{ng}$ of gerbera genomic DNA. PCR amplification was performed on a MasterCycler EP Gradient S (Eppendorf, Hamburg, Germany) with the following parameters: initial DNA template denaturation at $94{ }^{\circ} \mathrm{C}$ for $2 \mathrm{~min}$, followed by 5 cycles of $94{ }^{\circ} \mathrm{C}$ for $45 \mathrm{~s}, 40^{\circ} \mathrm{C}$ for $45 \mathrm{~s}$, and $72{ }^{\circ} \mathrm{C}$ for $1 \mathrm{~min}$, followed by 35 cycles of $94^{\circ} \mathrm{C}$ for $45 \mathrm{~s}, 53{ }^{\circ} \mathrm{C}$ for $45 \mathrm{~s}$, and $72{ }^{\circ} \mathrm{C}$ for $1 \mathrm{~min}$, and a final extension of $72{ }^{\circ} \mathrm{C}$ for $8 \mathrm{~min}$. PCR products were separated on a $6.5 \%$ polyacrylamide gel in $1 \times$ TBE buffer run at $1500 \mathrm{~V}$ for $4 \mathrm{~h}$ and visualized on a 4300 DNA Analyzer (LI-COR Biosciences, Lincoln, NE).

Genetic linkage and QTL analysis. Genetic linkage among the above-identified markers was determined by analyzing the markers' segregation in the 91 progeny of population 7-4. Marker analysis of the progeny was performed as described above. Two-point and multiple-point analysis of these markers was performed in MAPMAKER/EXP (Lander et al., 1987) using a logarithm (base (10) of odds (LOD) value threshold of 3.0. Genetic map distances among linked molecular markers 
were calculated in MAPMAKER/EXP with the "error detection function" on and using the Kosambi function. Linkage map data from MAPMAKER/EXP and the PM resistance phenotyping data were used as inputs for MAPMAKER/QTL to detect potential QTLs controlling PM resistance and to estimate the phenotypic effects of each QTL interval, as described by Lincoln et al. (1993).

POWDERY MILDEW SPORE GERMINATION, HYPHAL GROWTH, AND SPORE PRODUCTION. Powdery mildew conidia used for spore germination studies were produced on 'Sunburst Snow White' leaf segments maintained on $2 \%$ water agar. The fungal species causing PM on gerbera plants was identified as $P$. xanthii based on morphology of mycelium, conidia, conidiophore, and footcells, and rDNA sequence analysis (data not shown).

$P$. xanthii conidia were gently brushed onto gerbera leaf segments $(\approx 2 \mathrm{~cm}$ long by $\approx 1 \mathrm{~cm}$ wide) using a fine brush. The inoculated leaf segments were incubated in disposable plastic petri dishes $(9.5 \mathrm{~cm}$ in diameter) containing $20 \mathrm{~mL}$ of $2 \%$ water agar at $22{ }^{\circ} \mathrm{C}$, with a photoperiod of $16 \mathrm{~h}$ (light, $\approx 100$ $\mu \mathrm{mol} \cdot \mathrm{m}^{-2} \cdot \mathrm{s}^{-1}$ ) and $8 \mathrm{~h}$ (dark). Leaf segments were removed from the petri dishes at $12,18,24,48$, and $72 \mathrm{~h}$ post inoculation (hpi) and observed under a bright-field microscope (BH-2; Olympus, Center Valley, PA) to record spore germination, germ tube growth, appressorium formation, hyphal branching, and hyphal length. Conidia were considered germinated when the primary germ tube was at least half of the width of the conidia. For each time point in the above-described observations, three to five leaf segments from different leaves and at least 50 PM spores on each leaf segment were examined.

To count new conidia produced on inoculated gerbera leaves, pieces of leaf tissue (circles, $6 \mathrm{~mm}$ in diameter) were punched out of the inoculated leaves and immersed in $200 \mu \mathrm{L}$ of water with Tween 20 (0.05\%; Sigma-Aldrich, St. Louis, MO) in a $1.5-\mathrm{mL}$ microcentrifuge tube. These microcentrifuge tubes were placed on a mini-shaker (Vortex-Genie; Fisher Scientific, Waltham, MA) for 5-10 s to dislodge conidia from leaf surfaces; the conidia in the solution were then counted under a microscope (BH-2) with a hemocytometer. Conidium counts were converted into conidium densities (conidia per square centimeter of gerbera leaf surface). Conidium counting was performed every $2 \mathrm{~d}$, beginning $4 \mathrm{~d}$ post inoculation (dpi) and ending $12 \mathrm{dpi}$. For each time point of conidium counting, 10 to 15 replicates of leaf samples were examined.

Statiscal analysis. SAS ${ }^{\circledR}$ (version 9.2 for Windows; SAS Institute, Cary, NC) was used for analysis of variance (ANOVA) and correlation analysis of PM severity ratings. PM severity ratings were not transformed before ANOVA using the SAS ${ }^{\circledR}$ procedure Proc GLM or correlation analysis using the procedure Proc CORR. The computer software Microsoft ${ }^{\circledR}$ Excel $^{\circledR}$ (version 2010; Microsoft Corp., Redmond, WA) was used to calculate standard deviations for means of $P$. xanthii spore germination, germ tube and appressorium formation, and conidia density. Chi-square analysis was performed using the online tool "Calculation for the Chi-Square Test" (Preacher, 2001).

\section{Results}

Segregation of PM resistance among Progeny. In the PM evaluations, plants of UFGE 4033 had a PM severity rating between 1 and 3, whereas plants of 'Sunburst Snow White' had a PM severity rating between 9 and 10 . The PM severity rating among 90 progeny of UFGE 4033 and 'Sunburst Snow White' ranged from 2 to 10 in the first round of evaluation, 3 to 10 in the second round of evaluation, and 3 to 10 in the third round of evaluation, indicating substantial variation in PM resistance among the progeny.

The overall mean of PM severity ratings from the first round of evaluation (6.3) was lower than that from the third round of evaluation (7.2) at $P=0.0540$; the overall mean of PM severity ratings from the second round of evaluation (6.8) was not significantly different from that of the first and the third round of evaluation. Correlation among PM severity ratings of the three rounds of evaluations was highly significant $(P<0.0001)$, but Pearson correlation coefficients $(r)$ were only moderately high, 0.6051 to 0.6606 . PM severity ratings for about two-thirds of the progeny were relatively consistent (with rating differences no greater than 2 scales) from evaluation to evaluation, whereas PM severity ratings for about one-third of the progeny (33 out of 90) varied considerably (with differences greater than 2) from evaluation to evaluation. The average PM (PM-avg) severity rating for each progeny across three rounds of evaluation was better correlated with the ratings of individual evaluations (PM-0211, PM-0711, and PM-0112) $(r=0.8589$ to $0.8721, P<0.0001)$. Considering this, PM-avg values were used to represent the PM resistance phenotype of the progeny. PM-avg values in population 7-4 varied from 2.7 to 10.0 among progeny, and the distribution was continuous with two peaks at the 4.1-5.0 and the 7.1-8.0 intervals, respectively (Fig. 1), which suggests that the PM resistance trait in UFGE 4033 (and UFGE 31-19) is quantitative in nature and that one or more major genes may be responsible for PM resistance.

BSA AND MOlecular MaRkers PUTATIVEly Linked to PM RESISTANCE. Previously, we obtained 12 sequence characterized amplified region/cleaved amplified polymorphic sequence markers from cloned gerbera RGC sequences (Song et al., 2012). These markers were polymorphic between UFGE 4033

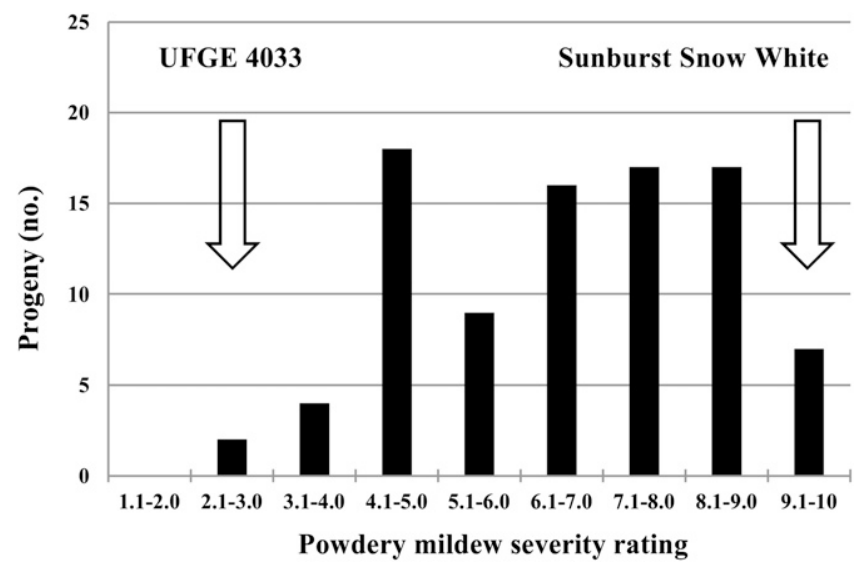

Fig. 1. Distribution of 90 progeny of the gerbera population 7-4 in relation to the powdery mildew (PM) severity ratings. The PM severity ratings were based on a scale of 1 to 10 as described by Hausbeck et al. (2002) with minor modifications, where $1=$ no disease, $2=1 \%$ to $10 \%, 3=11 \%$ to $20 \%, 4=21 \%$ to $30 \%, 5=31 \%$ to $40 \%, 6=41 \%$ to $50 \%, 7=51 \%$ to $60 \%, 8=61 \%$ to $70 \%$, $9=71 \%$ to $80 \%$, and $10=81 \%$ to $100 \%$ of the gerbera leaf area showing PM symptoms, and the PM severity ratings presented were the average from three rounds of evaluation conducted in Wimauma, FL, in Feb. 2011, July 2011, and Jan. 2012. UFGE 4033 (PM-resistant) and 'Sunburst Snow White' (PMsusceptible) are the female and male parents, respectively, of population 7-4, and their PM severity ratings are indicated by arrows. 


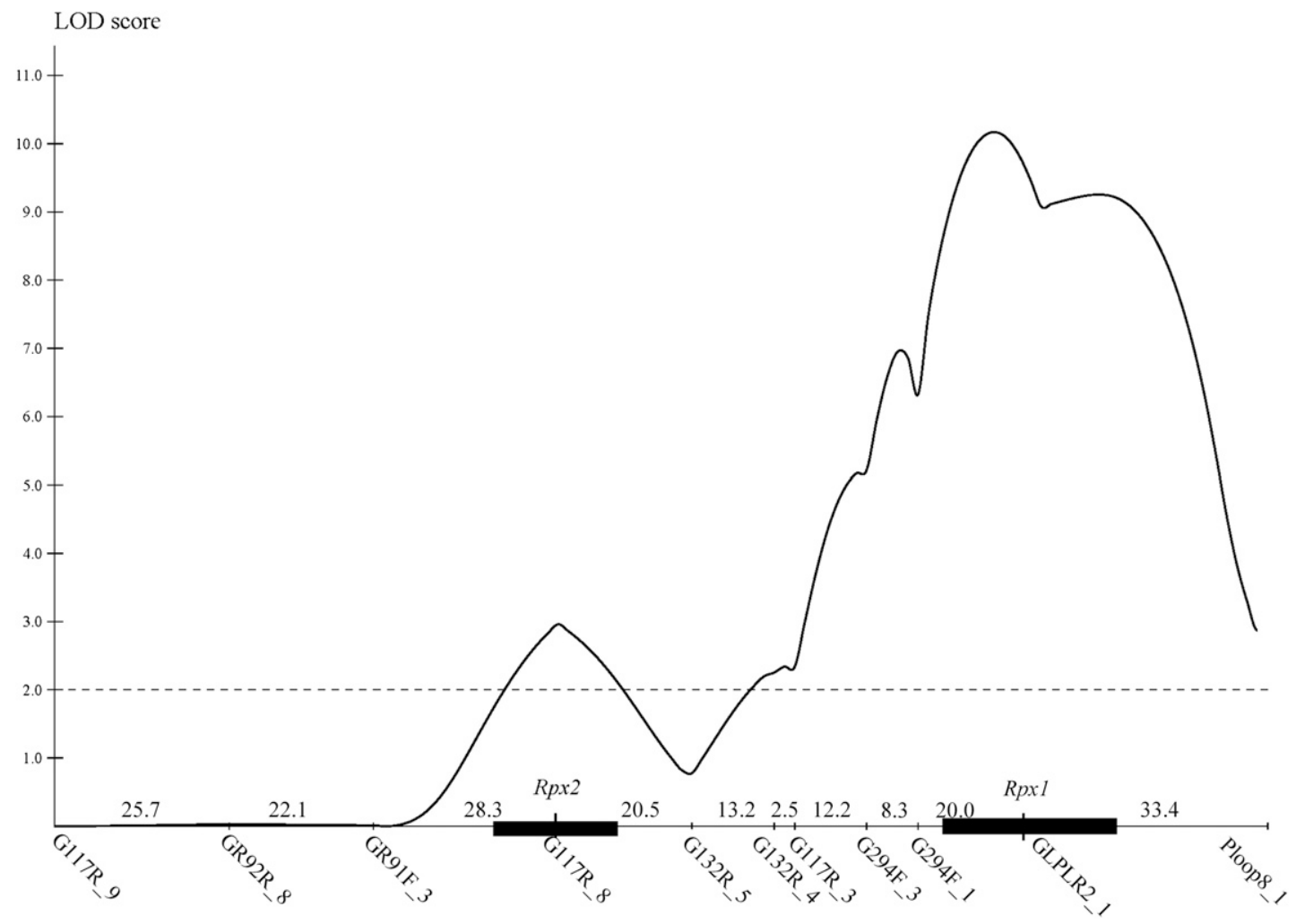

Fig. 2. Quantitative trait locus (QTL) mapping for powdery mildew (PM) resistance in gerbera breeding line UFGE 4033. The $x$-axis shows the genetic linkage map of gerbera linkage group 1 (LG1) calculated by MAPMAKER/EXP version 3.0b (Lander et al., 1987) using the Kosambi function; numbers immediately above the axis are genetic distances between adjacent markers in centiMorgans (cM). The y-axis shows the LOD [logarithm (base (10) of odds] scores of presence of QTLs for PM resistance calculated by MAPMAKER/QTL version 1.1b based on the average PM severity rating of three rounds of evaluations (PM-avg) in Wimauma, FL. The broken line above the x-axis indicates the default LOD threshold value used in detecting QTLs. The confidence intervals for QTLs RpxI and Rpx2 on the linkage map are indicated by shaded rectangles.

and 'Sunburst Snow White', but they did not detect polymorphisms between the PM-R and the PM-S bulks. Out of 99 gerbera EST-SSR markers screened, 13 were present in UFGE 4033 and absent in 'Sunburst Snow White', but none of them were polymorphic between the two DNA bulks. TRAP marker analysis identified 17,494 DNA fragments in UFGE 4033 and
'Sunburst Snow White' from 648 primer combinations. A total of 968 DNA bands were present in UFGE 4033 but absent in 'Sunburst Snow White', and 17 of these DNA bands were present in the PM-R bulk but absent in the PM-S bulk (Table 1). These DNA bands were considered as putative molecular markers linked to the PM resistance trait in UFGE 4033.

Table 1. Summary of bulked segregant analysis (BSA) using three marker systems to identify molecular markers present in the powdery mildew (PM)-resistant gerbera parent UFGE 4033 and the resistant DNA bulk but absent in the PM-susceptible parent 'Sunburst Snow White' and the susceptible DNA bulk.

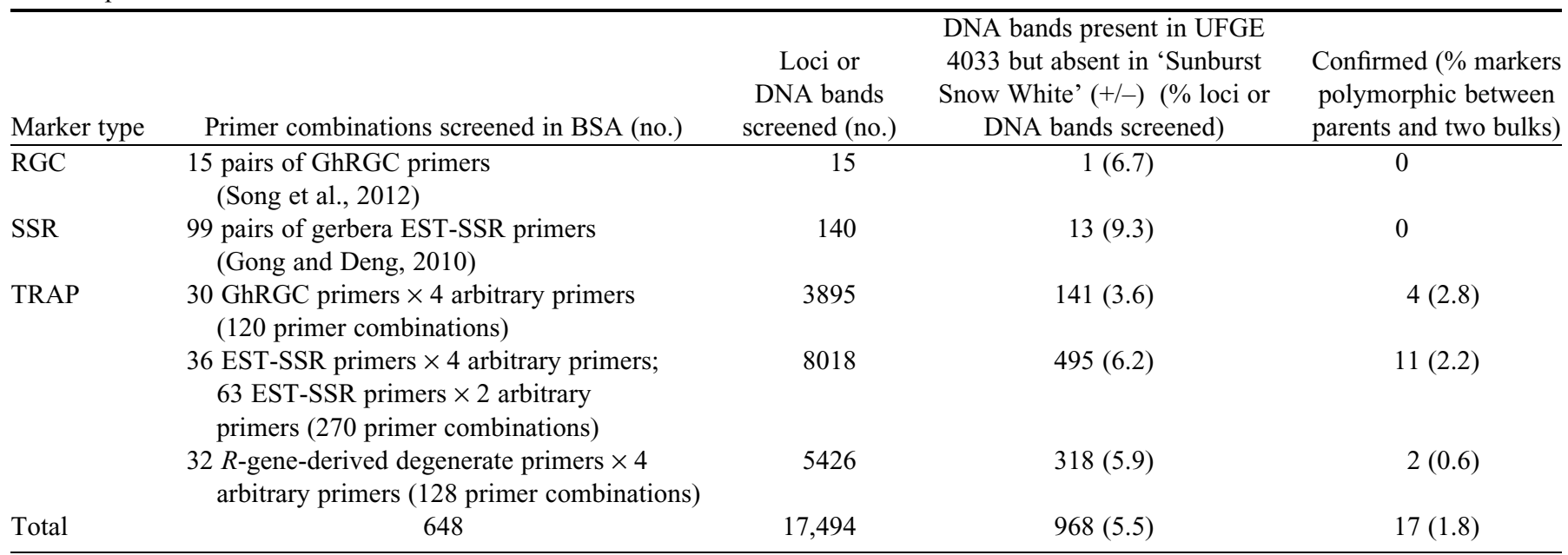


Table 2. Molecular markers mapped to Linkage Group 1 (LG1) and linked to quantitative trait loci for gerbera powdery mildew resistance, primers (and primer sequences) used to generate the markers, the approximate size of the polymorphic DNA fragments, and chi-square analysis of the segregation of the markers in gerbera population 7-4.

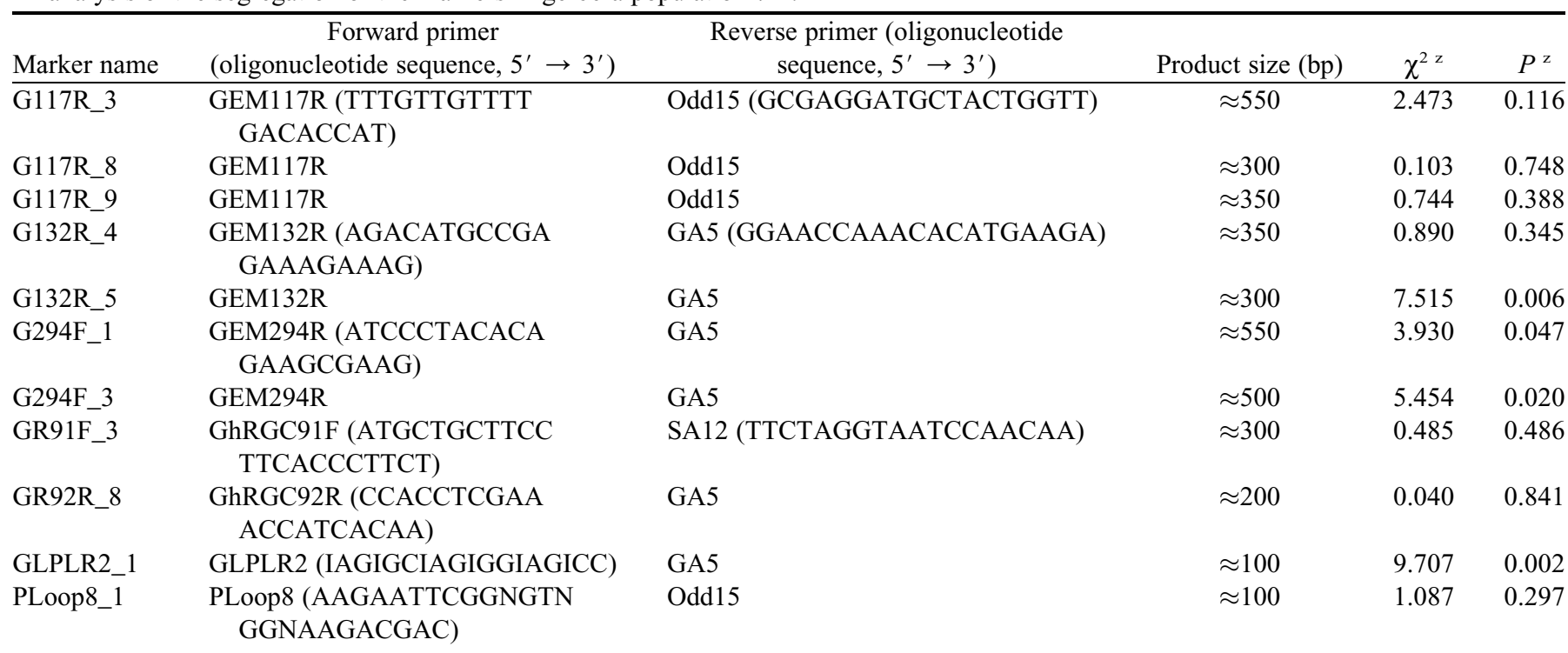

${ }^{\mathrm{z}}$ Chi-square tests were based on the theoretical 1:1 (presence: absence) segregation ratio expected for dominant markers in backcross populations and performed online (Preacher, 2001).

Genetic Linkage ANALYSIS ANd QTL identification. Ninety progeny of population 7-4 were genotyped for the 17 molecular markers. All 17 markers behaved as dominant markers, segregating as presence or absence of specific DNA bands. Twelve markers segregated in the expected 1:1 (present: absent) ratio, five markers exhibited skewed segregation (deviated from the expected $1: 1$ ratio) at $P \leq 0.05$, and two markers showed skewed segregation at $P \leq 0.10$.

Genetic linkage analysis using the MAPMAKER/EXP software separated 11 markers (G117R_3, G117R_8, G117R_9, G132R_4, G132R_5, G294F_1, G294F_3, GLPLR2_1, GR91F_3, GR92R_8, and PLoop8_1) into one linkage group [Linkage Group 1 (LG1)], two markers (G132R_1 and G132R_12) into another linkage group (LG2), and identified four markers (G132R_2, G296R_1, GR91F_7, and GR92R_12) as unlinked. The LG1 extended $186.1 \mathrm{cM}$, and the genetic distance between markers ranged from 2.5 to $33.4 \mathrm{cM}$ (Fig. 2). LG2 contained two markers that were tightly linked with a genetic distance of $1.2 \mathrm{cM}$. The primers and primer sequences used to amplify molecular markers in LG1 are presented in Table 2.

MAPMAKER/QTL analysis identified two QTLs in LG1 for PM-avg, the average PM severity ratings across three rounds of PM evaluation (Fig. 2). The interval between markers GLPLR2_1 and G294F_1 accounted for 56.5\% of the phenotypic variation in PM-avg and had an LOD value of 10.16, thus indicating a major QTL. The interval between markers G117R_8 and G132R_5 carried a QTL explaining 14.6\% of the phenotypic variation in PM-avg (LOD score $=2.96$ ).

When the linkage map was scanned against the PM-0211, PM-0711, and PM-0112 phenotyping data sets for QTLs, the first interval (the interval between markers GLPLR2_1 and G294_1) was detected in all scans, thus appearing to be a robust locus for PM resistance. We propose to name this major QTL as Rpxl (resistance to $\underline{P}$. $x$ anthii 1). The second interval (between markers G117R_8 and G132R_5) showed an LOD score between 2.54 and 3.10 when the linkage map was scanned against the PM-0211 and PM-07-11 phenotyping data sets; however, this interval fell below the MAPMAKER/QTL default LOD threshold value (2.0) and became undetected when the PM-0112 data set was used. Therefore, the significance of this interval toward PM resistance varied. Tentatively we name this interval as $R p x 2$. When $R p x 2$ was undetected in MAPMAKER/QTL, $R p x 1$ explained a greater percentage $(65.5 \%)$ of the observed phenotypic variance.

PATHOGEN SPORE Germination AND PROdUCTION. Inoculated $P$. xanthii conidia germinated readily on the leaf surface of 'Sunburst Snow White', with nearly $80 \%$ of the spores having germinated by 24 and 48 hpi (Fig. 3A). Spore germination after 48 hpi was difficult to determine reliably as new hyphae spread on the leaf surface. Appressoria were observed on primary germ tubes for nearly $50 \%$ of the germinated spores by $8 \mathrm{hpi}$ and for $80 \%$ of the germinated spores by 12 hpi (Fig. 3B). The percentage of germinated conidia forming appressoria remained high to the end of the observation period (48 hpi). Secondary germ tubes were not observed at $12 \mathrm{hpi}$, but $40 \%$ of the germinated spores produced secondary germ tubes by $18 \mathrm{hpi}$ (Fig. 3C). The percentage of germinated spores with secondary germ tubes reached $80 \%$ by 24 hpi and $90 \%$ by $48 \mathrm{hpi}$. Secondary hyphae began to appear by 48 hpi and increased their length rapidly thereafter (Fig. 3D). New conidia began to appear on inoculated leaves by $6 \mathrm{dpi}$; conidia density increased rapidly between 6 and 10 dpi (nearly 30-fold) and slightly between 10 and $12 \mathrm{dpi}(\approx 0.05$-fold $)$, reaching $111.4 \times 10^{3}$ conidia $/ \mathrm{cm}^{2}$ at 12 dpi (Fig. 4).

$P$. xanthii conidia germinated similarly well on the leaf surface of UFGE 4033 and PM-R progeny 1-25 (with a PM severity rating between 1 and 3 ), showed a similar trend of progression in germination, and reached a similarly high germination percentage $(80 \%)$ on the leaf surface of UFGE 

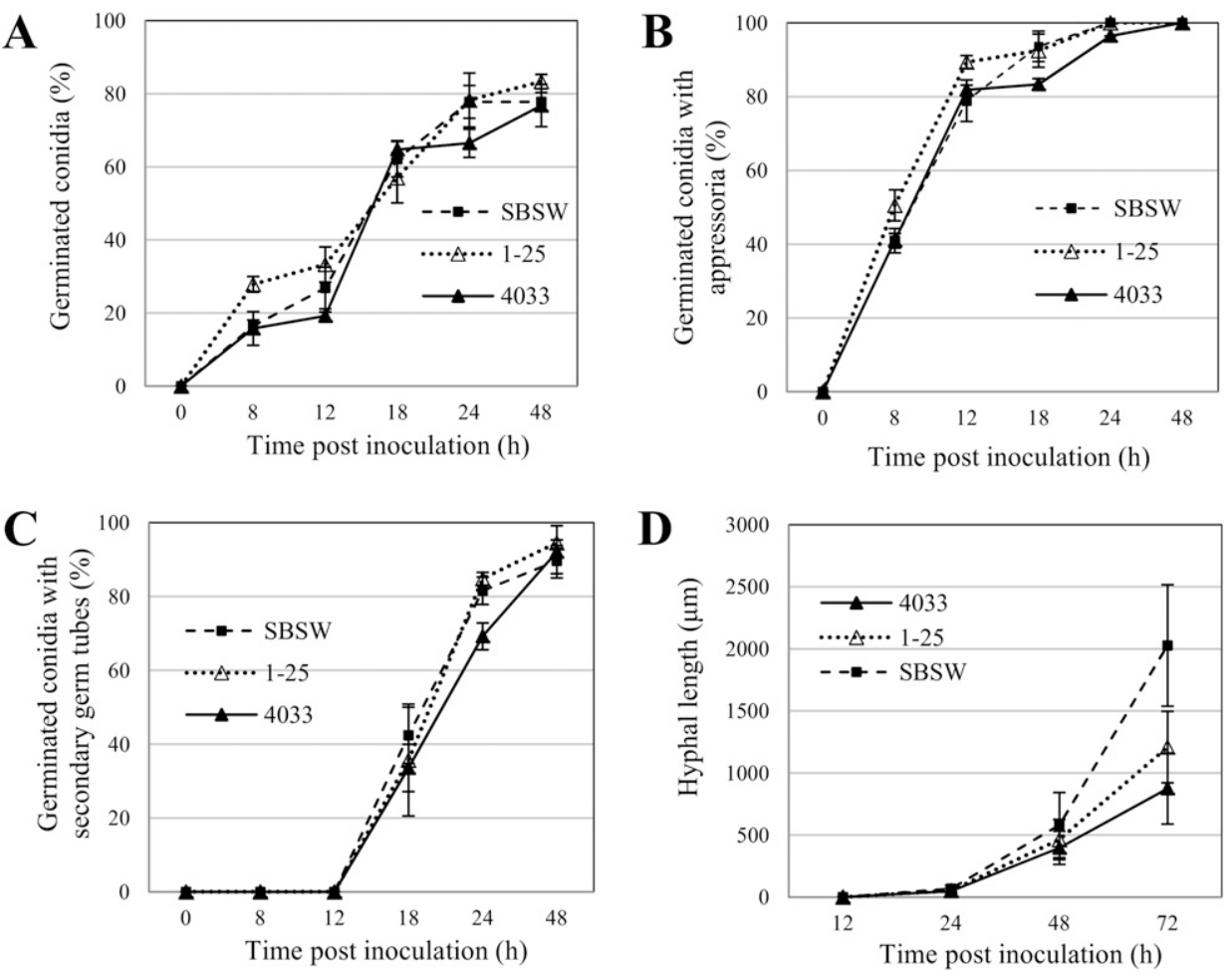

Fig. 3. (A) Conidial germination, (B) appressorium formation, (C) secondary germ tube formation, and (D) hyphal growth (length) of Podosphaera xanthii on the adaxial surface of leaf segments of gerbera UFGE 4033 [4033 (powdery mildew-resistant)], 'Sunburst Snow White' [SBSW (powdery mildew-susceptible)], and their progeny 1-25 [member of population 7-4 from a cross between UFGE 4033 and 'Sunburst Snow White (powdery mildew-resistant)] up to $72 \mathrm{~h}$ post inoculation. The inoculated gerbera leaf segments were maintained on $2 \%$ water agar inside sealed disposable petri dishes. Vertical bars indicate standard deviation.

4033 and progeny 1-25 (Fig. 3A). No significant differences were observed in appressorium or secondary germ tube formation on leaf surfaces of 'Sunburst Snow White' and UFGE 4033 or progeny $1-25$ at 12, 18, 24, and 48 hpi (Fig. 3B-C). However, differences were seen in hyphal branching, hyphal length, and, particularly, (new) conidia density.

Hyphal branching was not observed on the leaf surface of 'Sunburst Snow White', UFGE 4033, or progeny 1-25 at 24 hpi. Differences in hyphal branching began to emerge at 48 hpi and remained obvious at 72 hpi. $P$. xanthii colonies on 'Sunburst Snow White' leaves had an average of 0.95 and 5.5 hyphal branches per colony at 48 and $72 \mathrm{hpi}$, respectively. The fungal colonies on UFGE 4033 leaves had an average of 0.1 and 3.8 hyphal branches at 48 and 72 hpi, which were $89 \%$ and $31 \%$ reduced compared with 'Sunburst Snow White'. Similar levels of hyphal branching suppression were observed on the leaves of progeny 1-25. Hyphal branches were not counted after 72 hpi as the fungal colonies produced numerous branches and became difficult to count.

$P$. xanthii hyphae on UFGE 4033 leaves were much shorter than those on 'Sunburst Snow White' leaves. The longest hyphae in fungal colonies on UFGE 4033 leaves were $50 \mu \mathrm{m}$ at $48 \mathrm{hpi}$ and $110 \mu \mathrm{m}$ at $72 \mathrm{hpi}$, both being about $60 \%$ shorter than the longest hyphae on 'Sunburst Snow White' leaves $(120 \mu \mathrm{m}$ at $48 \mathrm{hpi}$ and $300 \mu \mathrm{m}$ at $72 \mathrm{hpi}$ ). The slower hyphal growth was also indicated by the total hyphal length per colony (Fig. 3D). At 72 hpi, the total length of hyphae per colony on UFGE 4033 leaves was $875 \mu \mathrm{m}, 56.7 \%$ shorter than the length of hyphae on 'Sunburst Snow White' leaves $(2027 \mu \mathrm{m})$ (Fig. 3D). Slower $P$.
'Sunburst Snow White' (Fig. 4).

xanthii hyphal growth was also observed on the leaves of progeny 1 25: the longest hyphal branches were $50 \%$ shorter at $48 \mathrm{hpi}$ and $30 \%$ shorter at $72 \mathrm{hpi}$, and the total length of hyphae per colony was $40 \%$ shorter at $72 \mathrm{hpi}$, compared with the corresponding measures on 'Sunburst Snow White' leaves (Fig. 3D).

Compared with 'Sunburst Snow White', P. xanthii conidia density on UFGE 4033 leaves was $79.7 \%$ lower at 6 dpi and greater than 95\% lower at 8, 10, and 12 dpi (Fig. 4). Similar degrees of spore density reduction $(91.5 \%$ to $94.2 \%)$ were recorded on the inoculated leaves of progeny $1-25$ at 8,10 , and $12 \mathrm{dpi}$ (Fig. 4). The spore densities on inoculated UFGE 4033 and progeny 1-25 leaves at 12 dpi were $5.1 \times 10^{3}$ and $9.4 \times 10^{3}$ conidia $/ \mathrm{cm}^{2}$, respectively. To confirm the suppression effects of PM resistance on spore production, another progeny individual (2-11) was inoculated and examined. This progeny showed a PM severity rating between 3 and 5 in the above-described PM resistance evaluations. On this progeny, $77 \%$ to $80 \%$ spore density reduction was observed compared with

\section{Discussion}

Information in the literature regarding the genetic bases of PM resistance in gerbera is scant. Kloos et al. (2005) reported a single dominant gene Pmrl controlling the resistance of gerbera plants 176 and 214 to $P$. xanthii. The authors observed that the dominance or expression of Pmrl varied with genetic background as well as gene dosage and suggested that other unidentified genes might modify Pmrl's effect on PM resistance phenotypes (Kloos et al., 2005). In that study, six PM resistance phenotype categories were defined (Kloos et al., 2005). Category R0 was very resistant with no colonies on any leaves; category R1 was resistant with $<20$ small colonies/leaf and no or light sporulation on a few leaves; and category R2 was moderately resistant with 20 to 30 colonies/leaf and light sporulation on many leaves. Categories S3, S4, and S5 were moderately susceptible, susceptible, and highly susceptible, respectively, with an increasing percentage of leaf area showing $\mathrm{PM}(\approx 50 \%$ to $75 \%$ for $\mathrm{S} 3,>75 \%$ for S4, and entire leaf surface for S5) and an increasing amount of sporulation (Kloos et al., 2005).

We applied these phenotype categories in this study but the resulting phenotype data (not shown) did not suggest a onegene model for the PM resistance in UFGE 4033, and the data were difficult to interpret. Therefore, we adopted another disease severity rating scale in evaluating gerbera PM severity (Hausbeck et al., 2002). Our PM severity ratings from three rounds of evaluation over nearly 15 months revealed continuous 


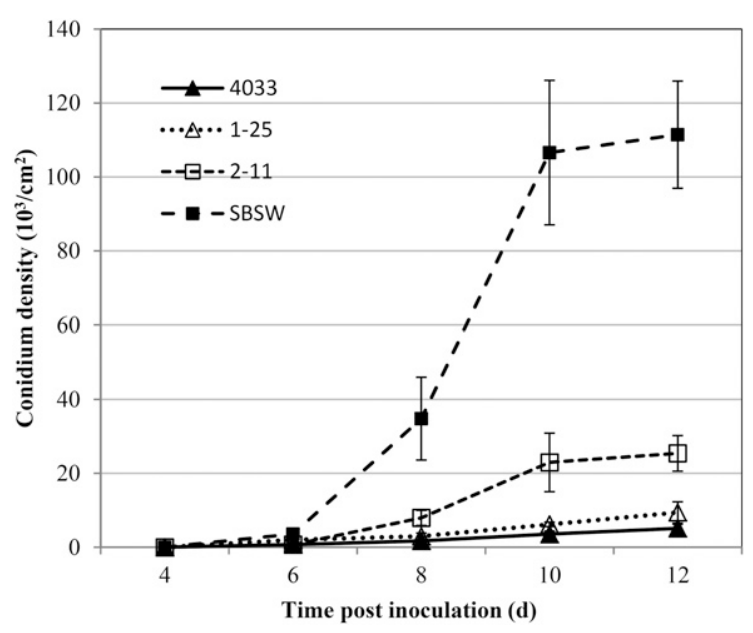

Fig. 4. Differences in Podosphaera xanthii spore (new conidium) density on the leaves of gerbera UFGE 4033 [4033 (powdery mildew-resistant)], 'Sunburst Snow White' [SBSW (powdery mildew-susceptible)], and their progeny 1-25 and 2-11 [members of population 7-4 (powdery mildew-resistant)]. Gerbera leaves were inoculated with $P$. xanthii conidia, maintained in vivo, and observed for production of new $P$. xanthii conidia.

variation from resistance to susceptibility among the progeny, which suggests the quantitative nature of the resistance in UFGE 4033 (and UFGE 31-19). QTL analysis identified a major QTL $(R p x 1)$ and a linked minor QTL (Rpx2) that together could explain more than $70 \%$ of the PM severity rating variance in the population 7-4.

The PM-R parent, UFGE 4033, developed typical PM symptoms in all three evaluations, though the symptoms were reduced. Thus, it may be more appropriate to classify the PM resistance in UFGE 4033 (and UFGE 31-19) as partial resistance. This notion of partial PM resistance seems supported by microscopic observation of the pathogen $P$. xanthii spore germination and infection of UFGE 4033 and 'Sunburst Snow White'. $P$ xanthii conida germinated equally well $(\approx 80 \%)$ on the leaf surface of UFGE 4033 and 'Sunburst Snow White', and a high percentage (approaching 100\%) of germinated spores formed appressoria and secondary germ tubes $(\approx 90 \%)$ on the leaf surface of UFGE 4033. The differences between UFGE 4033 and 'Sunburst Snow White' were that the former restricted $P$. xanthii hyphal growth and development, resulting in much shorter and less branched hyphae, and, particularly, reduced the production of new conidia. UFGE 4033's responses against $P$. xanthii, therefore, are quite different from hypersensitive responses that are often conditioned by race-specific resistance genes and involve rapid collapse of host cells and early arresting of PM spore germination and appressorium formation (Heath, 2000; Jørgensen, 1994; Xiao et al., 1997). UFGE 4033s responses to $P$. xanthii are similar to partial resistance that has been found in other PM pathosystems, including melon (Cucumis melo) and Sphaerotheca fuliginea (Kuzuya et al., 2003), and flowering dogwood (Cornus florida) and Erysiphe pulchra (Li et al., 2005). Although it may not present as strongly as complete resistance or immunity conferred by race-specific resistance genes, partial PM resistance may be effective against more pathogen races and over longer periods of time (more durable), which can be very useful and valuable for development and deployment of PM resistance and PM-R cultivars for commercial crop production (Jørgensen, 1994; Miedaner and Flath, 2007).
Genetic studies have revealed that multiple modes of inheritance may exist in plants for PM resistance traits. For example, six kinds of disease resistance have been found in barley (Hordeum vulgare) against PM caused by Blumeria graminis f.sp. hordei (previously Erysiphe graminis f.sp. hordei) (Jørgensen, 1994). More than 10 loci confer racespecific, broad-spectrum, or partial resistance to barley PM, and some loci (e.g., Mla and Mlo) each may have dozens of resistance alleles among different barley accessions (Jørgensen, 1994). Multiple loci have been identified for PM resistance in other plants, including melon against $P$. xanthii (Fukino et al., 2008), cucumber (Cucumis sativus) against $P$. fusca (formerly Sphaerotheca fuliginea) (He et al., 2013), and Arabidopsis thaliana against Erysiphe cruciferarum and E. cichoraceaum (Adam and Somerville, 1996; Xiao et al., 1997). Conceivably, different gerbera accessions may carry different resistance loci or alleles for $P$. xanthii. Other factors that might have led to the different conclusion between the present and the previous study (Kloos et al., 2005) about the genetic base of gerbera PM resistance may include existence of race specificities in $P$. xanthii causing PM on gerbera, and differences in the environment and growing conditions under which segregating gerbera populations were evaluated. Physiological races are known for PM fungal species (Fukino et al., 2008; Jørgensen, 1994); however, this subject has not been investigated in gerbera. Environmental and growing conditions are known to affect PM infection and severity in gerbera (Granke et al., 2012). Future studies may consider crossing various gerbera sources possessing PM resistance (e.g., gerbera plants 176 and 214, and UFGE 31-19 or UFGE 4033) with a common set of PM-S gerberas, evaluating progeny at the same sites under the same conditions, and using molecular markers to tag and map genes underlying the PM resistance. Such studies will be extremely valuable for understanding the genetic relationships among Pmrl, Rpxl, and $R p x 2$. The results might also be useful for refining breeding strategies for further improving gerbera PM resistance and developing new PM-R gerbera cultivars.

Like rust fungi, PM fungi are obligate, biotrophic pathogens and rely on special feeding structures, haustoria, to extract water and nutrients from host cells and to secrete pathogen effector proteins into host cells (Ma and Shang, 2009; Schulze-Lefert and Panstruga, 2003). When wind-blown PM spores germinate on the surface of susceptible leaves and other organs, germinating spores produce a germ tube ending with an appressorium. The appressorium forms a penetration peg to break through the cell wall of the host plant's epidermal cells and develop a haustorium by invaginating the host cell's plasma membrane. Proper formation and sustained functioning of haustoria are vital for PM fungi to infect and colonize host plants (Schulze-Lefert and Vogel, 2000). Frequent failure and/or malfunction of haustoria can lead to slow hyphal growth and reduced sporulation. It will be very interesting to determine if, and how, the formation, structure, and functioning of $P$. xanthii's haustoria are impacted in UFGE 4033, compared with 'Sunburst Snow White'. Histochemical and ultrastructural studies are needed to address this question.

Among the few sources of PM resistance identified so far in gerbera, UFGE 31-19 and its progeny UFGE 4033 are the only ones whose resistance has been successfully introduced into new gerbera cultivars and provide high levels of $\mathrm{PM}$ resistance in new cultivars (Deng and Harbaugh, 2010, 2013). This study has shown that the resistance in these gerberas is largely controlled by two linked QTLs and that the primary PM 
resistance mechanism in these gerberas is through restricting fungal growth and sporulation. These results lay a sound foundation for future use of this source of PM resistance in gerbera breeding. This study represents the first effort to identify and tag QTLs for disease resistance traits in gerbera. Genetically characterized gerbera genotypes UFGE 31-19 and UFGE 4033, in combination with gerbera plants 176 and 214 from a previous study (Kloos et al., 2005), could be essential plant materials for future investigation of possible race differentiation in $P$. xanthii, the causal agent of gerbera PM.

\section{Literature Cited}

Adam, L. and S.C. Somerville. 1996. Genetic characterization of five powdery mildew disease resistance loci in Arabidopsis thaliana. Plant J. 9:341-356.

Braun, U., R.T.A. Cook, A.J. Inman, and H.D. Shin. 2002. The taxonomy of the powdery mildew fungi, p. 13-55. In: R.R. Belanger, W.R. Bushnell, A.J. Dik, and T.L.W. Carver (eds.). The powdery mildews: A comprehensive treatise. APS Press, St. Paul, MN.

Channel, C. 2005. Gerbera trials rate plants. GM Pro 25:30-31.

Chung, Y., H. Kim, K. Kim, S. Park, Y. Yi, J. Lee, and O. Kwon. 2001. Morphological characteristics and genetic variation of gerbera (Gerbera hybrida Hort). J. Plant Biotechnol. 3:145-149.

Collins, N.C., N. Collins, C.A. Webb, S. Seah, and J.G. Ellis. 1998. The isolation and mapping of disease resistance gene analogs in maize. Mol. Plant Microbe Interactions 11:968-978.

Deng, Z. and B.K. Harbaugh. 2010. UFGE 4141, UFGE 7014, UFGE 7015, UFGE 7023, UFGE 7032, and UFGE 7034: Six new gerbera cultivars for marketing flowering plants in large containers. HortScience 45:971-974.

Deng, Z. and B.K. Harbaugh. 2013. UFGE 7031 and UFGE 7080 gerbera cultivars. HortScience 48:659-663.

Deng, Z., S. Huang, P. Ling, C. Chen, C. Yu, C.A. Weber, G.A. Moore, and F.G. Gmitter, Jr. 2000. Cloning and characterization of NBSLRR class resistance-gene candidate sequences in citrus. Theor. Appl. Genet. 101:814-822.

Doyle, J.J. and J.L. Doyle. 1987. A rapid DNA isolation procedure for small quantities of fresh leaf tissue. Phytochem. Bul. 19:11-15.

Ellwood, S., L. Kamphuis, T. Pfaff, R. Oliver, D. Samac, B.D. FosterHartnett, B. Tivoli, C. Onfroy, A. Moussart, A.M. Villegas, J.C. Sillero, and D. Rubiales. 2007. Medicago truncatula Handbook: Inoculation and Growth with Foliar Pathogenic Fungi. 12 Jan. 2011. $<$ https://www.noble.org/Global/medicagohandbook/pdf/Inoculation Growth_FoliarPathogenic.pdf>.

Evans, A. 2009. High Ranking Gerbera. 3 July 2013. <http://www. floraculture.nl/index.php?option=com_content\&view $=$ article\&id= 887: high-ranking-gerbera\&catid=65:february-2009\&Itemid=329>.

Fukino, N., T. Ohara, A.J. Monforte, M. Sugiyama, Y. Sakata, M. Kunihisa, and S. Matsumoto. 2008. Identification of QTLs for resistance to powdery mildew and SSR markers diagnostic for powdery mildew resistance genes in melon (Cucumis melo L.). Theor. Appl. Genet. doi: 10.1007/s00122-008-0885-1.

Gong, L. and Z. Deng. 2010. EST-SSR markers for gerbera (Gerbera hybrida). Mol. Breed. doi: 10.1007/s11032-009-9380-x.

Granke, L.L., L.E. Crawford, and M.K. Hausbeck. 2012. Factors affecting airborne concentrations of Podosphaera xanthii conidia and severity of gerbera powdery mildew. HortScience 47:1068-1072.

Hansen, H.V. 1999. A story of the cultivated Gerbera. New Plantsman 6:85-95.

Hausbeck, M.K. 2004. Take a long-range approach to powdery mildew resistance. GMPro 24:68-69.

Hausbeck, M.K., W.R. Quackenbush, and S.D. Linderman. 2002. Evaluation of cultivars of African daisy for resistance to powdery mildew. B\&C Tests 18:O0004.

He, X., Y. Li, S. Pandey, B.S. Yandell, M. Pathak, and Y. Weng. 2013. QTL mapping of powdery mildew resistance in WI2757 cucumber
(Cucumis sativus L.). Theor. Appl. Genet. doi: 10.1007/s00122-0132125-6.

Heath, M.C. 2000. Hypersensitive response-related death. Plant Mol. Biol. 44:321-334.

$\mathrm{Hu}$, J. and B.A. Vick. 2003. Target region amplification polymorphism: A novel marker technique for plant genotyping. Plant Mol. Biol. Rpt. 21:1-6.

Hu, J., O.E. Ochoa, M.J. Truco, and B.A. Vick. 2005. Application of TRAP technique to lettuce (Lactuca sativa L.) genotyping. Euphytica 144:225-235.

Jørgensen, J.H. 1994. Genetics of powdery mildew resistance in barley. Crit. Rev. Plant Sci. 13:97-119.

Kloos, W.E., C.G. George, and L.K. Sorge. 2005. Inheritance of powdery mildew resistance and leaf macrohair density in Gerbera hybrida. HortScience 40:1246-1251.

Kuzuya, M., K. Hosoya, K. Yashiro, K. Tomita, and H. Ezura. 2003. Powdery mildew (Sphaerotheca fuliginea) resistance in melon is selectable at the haploid level. J. Expt. Bot. 54:1069-1074.

Lander, E.S., P. Green, J. Abrahamson, A. Barlow, M.J. Daly, S.E. Lincoln, and L. Newburg. 1987. MAPMAKER: An interactive computer package for constructing primary genetic linkage maps of experimental and natural populations. Genomics 1:174-181.

Li, Y., M.T. Windham, R.N. Trigiano, D.C. Spiers, and W.E. Copes. 2005. Spore germination, infection structure formation, and colony development of Erysiphe pulchra on dogwood leaves and glass slides. Plant Dis. 89:1301-1304.

Lincoln, S.E., M.J. Daly, and E.S. Lander. 1993. Mapping genes controlling quantitative traits using MAPMAKER/QTL version 1.1: A tutorial and reference manual. Whitehead Institute, Cambridge, MA.

Ma, Q. and H.S. Shang. 2009. Ultrastructure of stripe rust (Puccinia striiformis f. sp. tritici) interacting with slow-rusting, highly resistant, and susceptible wheat cultivars. J. Plant. Pathol 91:597-606.

Mata, T.L.D., M.I. Segeren, A.S. Fonseca, and C.A. Colombo. 2009. Genetic divergence among gerbera accessions evaluated by RAPD. Scientia Hort. 121:92-96.

Michelmore, R.W., I. Paran, and R.V. Kesseli. 1991. Identification of markers linked to disease-resistance genes by bulked segregant analysis: A rapid method to detect markers in specific genomic regions by using segregating populations. Proc. Natl. Acad. Sci. USA 88:9828-9832.

Miedaner, T. and K. Flath. 2007. Effectiveness and environmental stability of quantitative powdery mildew (Blumeria graminis) resistance among winter wheat cultivars. Plant Breed., doi: 10.1111/ j.1439-0523.2006.01353.x.

Moyer, C. and N.A. Peres. 2012. Powdery mildew of gerbera daisy. 15 Jan. 2012. <http://edis.ifas.ufl.edu/pp257>.

Preacher, K.J. 2001. Calculation for the chi-square test: An interactive calculation tool for chi-square tests of goodness of fit and independence (computer software). 15 June 2013. <http://www.quantpsy.org/>.

Rogers, M.N. and B.O. Tjia. 1990. Gerbera production. Timber Press, Portland, OR.

Schulze-Lefert, P. and R. Panstruga. 2003. Establishment of biotrophy by parasitic fungi and reprogramming of host cells for disease resistance. Annu. Rev. Phytopathol. 41:641-667.

Schulze-Lefert, P. and J. Vogel. 2000. Closing the ranks to attach by powdery mildew. Trends Plant Sci. 5:343-348.

Song, X., Z. Deng, L. Gong, J. Hu, and Q. Ma. 2012. Cloning and characterization of resistance gene candidate sequences and molecular marker development in gerbera (Gerbera hybrida). Scientia Hort. 145:68-75.

Teeri, T.H., P. Elomaa, M. Kotilainen, and V.A. Albert. 2006. Mining plant diversity: Gerbera as a model system for plant developmental and biosynthetic research. Bioessays 28:756-767.

U.S. Department of Agriculture. 2013. Floriculture crops 2012 summary (April 2013). 25 Apr. 2013. < http://usda01.library.cornell. edu/usda/current/FlorCrop/FlorCrop-04-25-2013.pdf>.

Xiao, S., S. Ellwood, K. Findlay, R.P. Oliver, and J.G. Turner. 1997. Characterization of three loci controlling resistance of Arabidopsis thaliana accession Ms-0 to two powdery mildew diseases. Plant J. 12:757-768. 